\title{
Amostragem e agrupamento de dados de relação hipsométrica em inventários florestais de Cerrado Tocantinense
}

\author{
Valdir Carlos Lima de Andrade ${ }^{1 *}$, Elaine Aparecida Kroetz ${ }^{2}$, Andrei Nicola ${ }^{3}$, Priscila Bezerra de Souza ${ }^{1}$, Fabiano Kenji Nohama ${ }^{1}$, \\ Helio Garcia Leite ${ }^{4}$, Daniel Henrique Breda Binoti ${ }^{4}$, Mayra Luiza Marques da Silva Binoti ${ }^{5}$ \\ ${ }^{1}$ Universidade Federal do Tocantins, Rua Badejós, chácaras 69 e 72, Lt.07, Zona Rural, C. P. 66, CEP 77404-970, Gurupi,TO, Brasil \\ ${ }^{2}$ Engetec Engenharia Ltda, Rua Vereador Falcão Coelho, n’ 1645, CEP 77807-290, Araguaína, TO, Brasil \\ ${ }^{3}$ Engenheiro Florestal, Rua 14, n 112, CEP 77710-000, Pedro Afonso, TO, Brasil \\ ${ }^{4}$ Universidade Federal de Viçosa, Avenida Peter Henry Rolfs, s/n, Campus Universitário, CEP 36570-900, Viçosa, MG, Brasil \\ ${ }^{5}$ Universidade Federal do Espírito Santo, Av. Governador Lindemberg, 316 ,Centro, CEP 29550-000, Jerônimo Monteiro, ES, Brasil
}

"Autor correspondente:

vclandradeuft@gmail.com

Termos para indexação:

Cerrado tocantinense

Modelos hipsométricos

Identidade de modelos

Index terms:

Savanna tocantinense

Hypsometric models

Models identity

\section{Histórico do artigo:}

Recebido em 30 mar 2014

Aprovado em 18 ago 2015

Publicado em 30 set 2015

doi: 10.4336/2015.pfb.35.83.683
Resumo - Este estudo foi conduzido com o objetivo de avaliar o emprego de equações hipsométricas em cerrado sensu stricto focalizando o sistema de amostragem e o agrupamento dos dados de relação hipsométrica. Para isso, foram utilizadas 46 parcelas de $1.000 \mathrm{~m}^{2}$ cada, onde 13 parcelas foram empregadas na simulação de seis diferentes sistemas de amostragem e na estratificação de dados para o ajuste de modelos hipsométricos. As 33 parcelas restantes foram reservadas para um teste de aplicação das equações hipsométricas geradas, onde, para decidir quanto ao modelo hipsométrico e sistema de amostragem, foram adotados os critérios estatísticos: erro padrão residual e correlação linear múltipla, além da análise da distribuição de resíduos. Após esta decisão, foi utilizado um teste de identidade de modelos para verificar a melhor forma de preparar a base de dados visando o ajuste de modelos hipsométricos. Concluiu-se que a relação funcional linear: $\operatorname{Ln}(\mathrm{h})=\mathrm{f}[\operatorname{Ln}(\mathrm{N}) ; \operatorname{Ln}(\mathrm{dq} / \mathrm{d}) ; 1 / \mathrm{d}]$ foi a mais adequada para caracterizar a relação hipsométrica em inventários florestais de cerrado sensu stricto e deve ser ajustada com dados obtidos nas 50 primeiras árvores de cada parcela, sem agrupamento por classe de diâmetro.

\section{Sampling and stratification hypsometric relation data in forest inventories of savanna}

Abstract - This study aimed at evaluating the application of linear hypsometric equations in savanna located in the state of Tocantins, Brazil. Data were obtained in 46 plots of $1,000 \mathrm{~m}^{2}$ each, and 13 plots were used to simulate six different systems of data sampling to fit hypsometric models. The remaining 33 plots were reserved for an application test of generated hypsometric equations. In this assessment, to decide the sampling system and model, in addition to the residual graphic analysis, the following statistic criteria were adopted: residual standard error and multiple linear correlation. After this decision, a model identity test was applied to determine the best way to prepare the database aiming at the hypsometric models adjustment. We concluded that the linear functional relation: $\operatorname{Ln}(\mathrm{h})=\mathrm{f}[\operatorname{Ln}(\mathrm{N}) ; \operatorname{Ln}(\mathrm{dq} / \mathrm{d}) ; 1 / \mathrm{d}]$ fitted with data from the first 50 trees of each plot and, independently, from diameter class, was the most appropriate to characterize the hypsometric relation in savanna in Tocantins. 


\section{Introdução}

O conhecimento do estoque de madeira em povoamentos florestais, expresso pelo seu volume, é essencial para orientar decisões visando atender demandas de determinados produtos florestais de forma otimizada. Ocorre que o volume de madeira, na grande maioria das vezes, é obtido por meio de equações de dupla entrada, levando-se em conta o diâmetro e a altura das árvores. Esta, devido à sua medição ser onerosa, acarreta considerável aumento do custo de um inventário florestal.

Uma das alternativas para reduzir o custo do inventário, advindo da medição da altura de árvores, é medir esta variável em apenas parte das árvores que compõem as parcelas de inventário e, por meio de análise de regressão, gerar equações hipsométricas para obter a altura de árvores em que se mediu apenas o seu diâmetro. Neste tocante, observam-se muitos trabalhos no Brasil envolvendo, em sua maioria, situações caracterizadas em povoamentos florestais equiâneos com pinus (Schimidt, 1977; Cardoso et al., 1989; Machado et al., 1994, no Paraná, Barros et al., 2002 em São Paulo, Mendonça et al., 2011, em Minas Gerais); com eucalipto (Batista et al. 2001, em São Paulo; Leite \& Andrade, 2003 e Ribeiro et al., 2010, na Bahia); com araucária (Caldeira et al., 2003, no Paraná); com teca (Rossi et al., 2011, no Pará) e em plantios em Minas Gerais com 11 diferentes espécies nativas (Soares et al., 2011) e com candeia (Araújo et al., 2012), dentre muitos outros.

No entanto, devido a dificuldades na aplicação de equações hipsométricas em povoamentos florestais inequiâneos, como alto grau de heterogeneidade da relação altura e diâmetro, trabalhos sobre equações hipsométricas são pouco desenvolvidos no Brasil em comparação com florestas equiâneas, cabendo citar: Durlo \& Denardi (1998), em Floresta Estacional Decidual do Rio Grande do Sul com enfoque em Cabralea canjerana; Batista et al. (2001), em Floresta Tropical Equatorial Ombrófila Densa do Maranhão e em Mata Atlântica de diferentes locais do litoral de São Paulo, com enfoque em Tabebuia cassinoides; Bartoszeck et al. (2004), em bracatingais nativos no Paraná; Andrade et al. (2006), em Mata Atlântica secundária localizada no norte do Rio de Janeiro; Costa et al. (2012), com diferentes fisionomias de floresta em Minas Gerais com enfoque em Copaifera langsdorffi; e Machado et al. (2008) e Sanqueta et al. (2013), em fragmento de Floresta Ombrófila Mista no Paraná.
É importante ressaltar que o uso de relações hipsométricas requer basicamente que três atividades sejam realizadas para que se obtenha êxito na sua implementação num sistema de inventário florestal, avaliar diferentes modelos hipsométricos a fim de selecionar o mais adequado; avaliar diferentes sistemas de amostragem de dados de relação hipsométrica nas parcelas de inventário; e avaliar diferentes formas de estratificação destes dados para proceder ao ajuste do modelo hipsométrico selecionado. Estas etapas foram o foco de alguns estudos feitos em plantios florestais, como os trabalhos de Barros et al. (2002), Caldeira et al. (2003), Leite \& Andrade (2003), Ribeiro et al. (2010) e Araújo et al. (2012).

No entanto, a maioria dos estudos sobre relação hipsométrica em mata nativa focalizam apenas a avaliação de equações hipsométricas, sendo exceção, por exemplo, os trabalhos de Durlo \& Denardi (1998), Bartoszeck et al. (2004), Andrade et al. (2006) e Sanqueta et al. (2013). Nestes trabalhos houve, também, a preocupação em como os dados amostrados deveriam ser agrupados, ou estratificados, para ajuste de um modelo hipsométrico. No entanto, apenas em Andrade et al. (2006) é que se observa o foco na avaliação do sistema de amostragem de dados de relação hipsométrica em mata nativa.

Nota-se que trabalhos sobre equações hipsométricas em áreas de cerrado são inexistentes e, dada à importância deste Bioma, estudos envolvendo a forma de amostragem de dados de relação hipsométrica e o agrupamento ou não destes, se justificam a fim de tornar mais ágil o inventário e, consequentemente, promover redução de custos e melhorias no desempenho de modelos hipsométricos. Portanto, este trabalho teve por objetivo avaliar o emprego de equações hipsométricas em área de cerrado com foco em diferentes sistemas de amostragem da relação hipsométrica e de estratégias de formação da base de dados.

\section{Material e métodos}

\section{Área de estudo e sua caracterização}

Para o estudo de caso, foi utilizada uma área de cerrado sensu stricto localizada no município de Goiatins, Estado do Tocantins, próxima à divisa do Estado do Maranhão, Brasil. Nesta região, conforme Seplan (2008), o clima é classificado como B1wA'a', com evapotranspiração potencial e variação média anual de 1.400 a 1.700 mm, 
com precipitação média no verão em torno de 390 a 480 $\mathrm{mm}$ ao longo de três meses consecutivos.

A região tem solos concrecionários, que compreende solos minerais formados sob condições de restrição à percolação da água, sujeitos ao efeito temporário de excessiva umidade e expressiva plintização com ou sem petroplintita (Sistema..., 1999; Seplan, 2005). Sua cobertura vegetal apresenta predominância de cerrado sensu stricto (Atlas do Tocantins, 2008) a qual se caracteriza pela presença de árvores baixas, inclinadas, tortuosas, com ramificações irregulares e retorcidas. Os arbustos ou subarbustos encontram-se espalhados com algumas espécies apresentando órgãos subterrâneos perenes, que permitem a rebrota após a queima ou corte (Coutinho, 1978).

Vale ressaltar, que o Cerrado é o segundo maior bioma brasileiro, ocupando cerca de $21 \%$ do território nacional e cujo termo é utilizado para designar um conjunto de ecossistemas que ocorrem no Brasil Central (Eiten,
1977; Felfili \& Silva-Junior,1988; Fidelis \& Godoy, 2003; Assunção \& Felfili, 2004).

\section{Coleta de dados e análise}

Para ajuste e avaliação dos modelos hipsométricos (Tabela 1), foram utilizados dados obtidos em 46 parcelas de $1.000 \mathrm{~m}^{2}$ cada (faixas de $10 \mathrm{~m} \mathrm{x} 100 \mathrm{~m}$ ), distribuídas sistematicamente em uma área de cerrado sensu stricto tocantinense. Em todas as árvores das parcelas foi medida a circunferência a $1,30 \mathrm{~m}$ do solo (CAP) com fita métrica, e a altura total com régua. $\mathrm{O}$ CAP foi transformado em diâmetro (d) com posterior separação nas classes: $\mathrm{d} \leq 10 \mathrm{~cm}, 10 \mathrm{~cm}<\mathrm{d} \leq 15 \mathrm{~cm}$ e $\mathrm{d}>15 \mathrm{~cm}$. Os modelos 1, 2, 3 e 4 foram avaliados anteriormente em outras regiões no Brasil (Cardoso et al., 1989; Batista et al., 2001; Bartoszeck et al., 2004; Andrade et al., 2006; Machado et al., 2008; Ribeiro et al., 2010; Mendonça et al., 2011; Araújo et al., 2012; Costa et al., 2012; Sanqueta et al., 2013) e os demais $(5,6,7$, e 8$)$ são modelos hipsométricos desenvolvidos para este trabalho.

Tabela 1. Modelos lineares testados para gerar equações hipsométricas em uma área de cerrado sensu stricto, localizada na região nordeste do Estado do Tocantins, Brasil.

\begin{tabular}{|c|c|c|}
\hline Modelo & Forma funcional de ajuste & Forma funcional de uso \\
\hline 1 & $\operatorname{Ln}(h)=f\left(\frac{1}{d}\right)$ & $\hat{h}=\exp \left[f\left(\frac{1}{d}\right)\right.$ \\
\hline 2 & $\operatorname{Ln}(h)=f[\operatorname{Ln}(d)]$ & $\hat{h}=\exp \{f[\operatorname{Ln}(d)]\}$ \\
\hline 3 & $(h)=f[\operatorname{Ln}(d)]$ & $\hat{h}=f[\operatorname{Ln}(d)]$ \\
\hline 4 & $(h)=\frac{d^{2}}{f\left(d ; d^{2}\right)}$ & $\hat{h}=\frac{d^{2}}{f\left(d ; d^{2}\right)}$ \\
\hline 5 & $\operatorname{Lnh}=f[\operatorname{Ln}(G) ; \operatorname{Ln}(N) ; d]$ & $\hat{h}=\exp \{f[\operatorname{Ln}(G) ; \operatorname{Ln}(N) ;(d)]\}$ \\
\hline 6 & $\operatorname{Ln}(h)=f\left[\operatorname{Ln}(N) ; \operatorname{Ln}\left(\frac{d q}{d}\right) ; \frac{1}{d}\right.$ & $\hat{h}=\exp \left\{f\left[\operatorname{Ln}(N) ; \operatorname{Ln}\left(\frac{d q}{d}\right) ;\left(\frac{1}{d}\right)\right.\right.$ \\
\hline 7 & $h=f\left(d ; d^{2} ; N\right)$ & $\hat{h}=f\left[(d) ;(d)^{2} ;(N)\right]$ \\
\hline 8 & $h=f\left(d ; d^{2} ; G\right)$ & $\hat{h}=f\left[(d) ;(d)^{2} ;(G)\right]$ \\
\hline
\end{tabular}

em que: $\mathrm{h}=$ altura total $(\mathrm{m}), \mathrm{d}=$ diâmetro a $1,3 \mathrm{~m}$ do solo $(\mathrm{cm}), \mathrm{dq}=$ diâmetro médio quadrático $(\mathrm{cm}), \mathrm{N}=$ número de árvores por ha, $\mathrm{G}=$ área basal $\left(\mathrm{m}^{2} \mathrm{ha}^{-1}\right), \mathrm{Ln}=$ logaritmo neperiano e exp $=$ inverso de $\mathrm{Ln}$. 
Das 46 parcelas, 13 foram utilizadas no ajuste dos modelos hipsométricos (Tabela 1). Por meio de teste de aplicação, utilizando-se as outras 33 parcelas, avaliou-se o desempenho na predição da altura das árvores, cujos dados são independentes das 13 parcelas utilizadas para estimar equações hipsométricas. Nesta avaliação foram analisadas a distribuição de resíduos e as seguintes estatísticas:

$$
s(h \hat{h})= \pm\left[\frac{\sqrt{\sum_{i=1}^{n}\left(h_{i}-\hat{h_{i}}\right)^{2} /(n-2)}}{\bar{h}}\right] 100
$$

e

$$
r(h \hat{h})=\left[\frac{\sum_{i=1}^{n} h_{i} \hat{h}_{i}-\frac{\left(\sum_{i=1}^{n} h_{i}\right)\left(\sum_{i=1}^{n} \hat{h_{i}}\right)}{n}}{\sqrt{\left[\sum_{i=1}^{n}\left(h_{i}-\bar{h}\right)^{2}\right]\left[\sum_{i=1}^{n}\left(\hat{h_{i}}-\bar{h}\right)^{2}\right]}}\right] 100
$$

Em que: $s(h \hat{h})=$ erro padrão residual $(\%), r(h \hat{h})$ =correlação linear $(\%), h_{\mathrm{i}}$ e $\hat{\mathrm{h}}_{\mathrm{i}}=$ respectivamente, altura real e estimada/predita da $\mathrm{i}$-ésima árvore $(\mathrm{m}), \mathrm{n}=$ número de pares de $h_{i}$ e $\hat{h}_{\mathrm{i}}$ e $\hbar=$ média da altura real $(m)$.

Os modelos hipsométricos foram classificados em locais e regionais, dependendo da sua formulação de relacionamento funcional. Neste caso, uma relação hipsométrica local é função apenas de uma variável independente, na maioria dos casos o diâmetro. As relações hipsométricas regionais expressam a altura da árvore em função de mais de uma variável independente, utilizando-se além do diâmetro, variáveis do povoamento que, neste trabalho, foram: número de árvores, área basal e diâmetro médio quadrático. Assim, para o ajuste dos modelos hipsométricos locais e regionais, utilizou-se dados obtidos em seis diferentes sistemas de amostragem simulados com os dados das 13 parcelas, quais sejam:

1) Amostragem com cinco árvores por classe diamétrica (A5ACD): uso das cinco primeiras árvores que ocorreram em cada classe de diâmetro e parcela.

2) Amostragem com dez árvores por classe diamétrica (A10ACD): uso das dez primeiras árvores que ocorreram em cada classe de diâmetro e parcela.

3) Amostragem com quinze árvores por classe diamétrica (A15ACD): uso das quinze primeiras árvores que ocorreram em cada classe de diâmetro e parcela.

4) Amostragem com trinta árvores por parcela (A30AP): uso das trinta primeiras árvores que ocorreram em cada parcela, independentemente, da classe de diâmetro.

5) Amostragem com quarenta árvores por parcela (A40AP): uso das quarenta primeiras árvores que ocorreram em cada parcela, independentemente, da classe de diâmetro.

6) Amostragem com cinquenta árvores por parcela (A50AP): uso das cinquenta primeiras árvores que ocorreram em cada parcela, independentemente, da classe de diâmetro.

Após a decisão das equações hipsométricas locais e regionais de melhor desempenho, considerando o melhor sistema de amostragem para ajuste destes em cerrado sensu stricto, realizou-se análise com os dados das 13 parcelas, visando à formação da base de dados para estimar equações hipsométricas. Para este propósito, com uma significância de 5\%, foi utilizado o teste $\mathrm{F}_{\mathrm{H}(0)}$ conforme Regazzi (1996), Leite \& Andrade (2003) e Araujo et al. (2012). Assim, calculou-se a seguinte estatística:

$$
F_{(H o)}=\left[\frac{\operatorname{Redução~}\left(H_{0}\right)}{(H-1) p}\right]\left[\frac{S Q \operatorname{Res}(c)}{N-H p}\right]^{-1}
$$

E m q u e : Redução $\left(H_{0}\right)=\operatorname{SQPar}(c)-\operatorname{SQPar}(r)$, SQPar $(c)=$ soma de quadrados de parâmetros do modelo completo, SQPar(r) = soma de quadrados de parâmetros do modelo reduzido, SQRes(c) = soma de quadrados do resíduo aplicando a equação do modelo completo, $\mathrm{H}=$ número de equações comparadas, $\mathrm{P}=$ número de parâmetros do modelo reduzido e $\mathrm{N}=$ número total de observações considerando $\mathrm{H}$ equações estimadas.

$\mathrm{O}$ teste $\mathrm{F}_{(\mathrm{Ho})}$ foi utilizado para verificar a existência de igualdade ou diferença no banco de dados envolvendo as parcelas e as classes de diâmetro, isto é, para decidir se é mais apropriado usar todas as informações incluídas em um único lote de dados (modelo reduzido), ou separando os dados por parcela (modelo completo) ou classe de diâmetro (modelo completo). Assim, quando $\mathrm{F}_{(\mathrm{Ho})}$ resulta em não significância estatística é mais apropriado considerar as diferentes situações do banco de dados como um único lote de dados para estimar uma equação hipsométrica. Caso contrário, o mais apropriado é ajustar, separadamente, uma equação por parcela ou por classe de diâmetro. 


\section{Resultados e discussão}

As 13 parcelas utilizadas para ajuste dos modelos (Tabela 1) totalizaram 1.491 árvores, das quais foram utilizadas $195(13,1 \%)$ no sistema de amostragem A5ACD, 390 (26,2\%) no sistema A10ACD, 585 (39,2\%) no sistema A15ACD, 390 (26,2\%) no sistema A30AP, $520(34,9 \%)$ no sistema A40AP e $650(43,6 \%)$ no sistema A50AP.

Os resultados dos critérios estatísticos obtidos no teste de aplicação com as 33 parcelas reservadas para este fim, com um total de 2.229 árvores, permitiram evidenciar superioridade dos modelos locais 1 e 4 em relação aos modelos 2 e 3, sendo o modelo 1 superior apenas no sistema A5ACD. Quanto aos demais modelos, observouse os piores resultados para o modelo regional 5. O modelo regional 7 , por ser superior ao modelo regional 8 , permitiu inferir que se deve incluir $\mathrm{N}$ no lugar de $\mathrm{G}$ na relação funcional da altura com a forma quadrática do diâmetro, isto é, para cerrado sensu stricto tocantinense é preferível ajustar a forma funcional linear $\mathrm{h}=\mathrm{f}\left(\mathrm{d} ; \mathrm{d}^{2} ; \mathrm{N}\right)$ ao invés de $\mathrm{h}=\mathrm{f}\left(\mathrm{d} ; \mathrm{d}^{2} ; \mathrm{G}\right)$.

Por outro lado, na análise da distribuição dos resíduos, verificou-se um melhor comportamento do modelo local 1 em relação ao 4 . Cabe ressaltar que o modelo regional 6 teve o melhor comportamento dos resíduos dentre todos os modelos lineares avaliados. Portanto, para gerar equações hipsométricas em cerrado sensu stricto do Tocantins, as formas funcionais $\operatorname{Ln}(h)=f\left(\frac{1}{d}\right)$ e

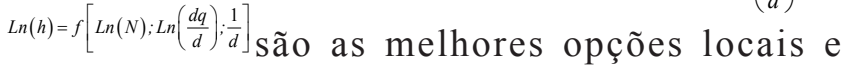
regionais, respectivamente.
Devido aos resultados de $\mathrm{s}(\mathrm{h} \hat{\mathrm{h}})$ e $\mathrm{r}(\mathrm{h} \hat{\mathrm{h}})$ serem muito próximos entre os diferentes sistemas de amostragem e modelos hipsométricos (Tabela 2), selecionou-se os sistemas A5CD e A50AP, cuja distribuição de resíduos mostra o modelo 6 com distribuição mais uniforme e equilibrada em relação ao modelo 1 , com a maioria dos resíduos se dispersando em $\pm 2 \mathrm{~m}$ (Figuras $1 \mathrm{~b}$ e $1 \mathrm{f}$ versus $1 \mathrm{a}$ e 1e). Vale ressaltar a superioridade do modelo 6 , com a maioria dos resíduos oscilando em $\pm 20 \%$, ou seja, tendo o melhor histograma de resíduos percentuais (Figuras $1 \mathrm{~d}$ e $1 \mathrm{~h}$ versus $1 \mathrm{c}$ e $1 \mathrm{~g}$ ). Assim, considerandose apenas o modelo 6 , nota-se melhor distribuição dos resíduos para o sistema A50AP (Figura 1e versus $1 \mathrm{fe} 1 \mathrm{~g}$ versus $1 \mathrm{~h}$ ). Portanto, dentre oito modelos hipsométricos ajustados para seis sistemas de amostragem, o modelo 6 é o mais adequado para gerar equações hipsométricas em áreas de cerrado tocantinense empregando-se o sistema A50AP.

A fim de complementar o exposto anteriormente, elaborou-se a Figura 2 com quatro parcelas selecionadas ao acaso, usando-se a base de dados com 13 parcelas. Nota-se que o sistema A50AP caracterizou melhor a dispersão dos dados de relação hipsométrica que o sistema A5ACD. Isto é essencial para reduzir o viés advindo da não medição da altura de todas as árvores das parcelas em um inventário florestal. Portanto, dentre os seis sistemas de amostragem da relação hipsométrica simulados neste trabalho, complementa-se a análise anterior sobre ser mais apropriado amostrar a altura das 50 primeiras árvores de cada parcela de inventário em áreas de cerrado sensu stricto tocantinense. 
Tabela 2. Estatísticas obtidas para os modelos 1 e 6 em seis sistemas de amostragem de relação hipsométrica simulados numa área de cerrado sensu stricto tocantinense.

\begin{tabular}{rrrrrrrr}
\hline \multirow{2}{*}{ SA } & MODELO & $\hat{\mathrm{b}_{0}}$ & $\hat{\mathrm{b}_{1}}$ & $\hat{\mathrm{b}_{2}}$ & $\hat{\mathrm{b}_{3}}$ & $\mathrm{~s}(\mathrm{~h} \hat{\mathrm{h}}) \%$ & $\mathrm{r}(\mathrm{hh}) \%$ \\
\hline A5ACD & 1 & 2,366 & $-5,839$ & - & - & 30,54 & 71,43 \\
& 6 & 0,692 & 0,240 & 0,022 & $-6,064$ & 29,04 & 75,83 \\
A10ACD & 1 & 2,357 & $-5,706$ & - & - & 30,70 & 71,41 \\
& 6 & 0,926 & 0,180 & $-0,178$ & $-4,007$ & 28,25 & 76,04 \\
A15ACD & 1 & 2,351 & $-5,737$ & - & - & 30,49 & 71,41 \\
& 6 & 0,877 & 0,184 & $-0,190$ & $-3,918$ & 28,35 & 76,05 \\
A30AP & 1 & 2,354 & $-5,595$ & - & - & 30,95 & 71,38 \\
& 6 & 0,360 & 0,275 & $-0,113$ & $-4,988$ & 28,49 & 76,12 \\
A40AP & 1 & 2,314 & $-5,349$ & - & - & 30,77 & 71,33 \\
& 6 & 0,366 & 0,260 & $-0,198$ & $-4,026$ & 28,63 & 75,95 \\
A50AP & 1 & 2,311 & $-5,348$ & - & - & 30,71 & 71,33 \\
& 6 & 0,441 & 0,244 & $-0,228$ & $-3,666$ & 28,58 & 75,89 \\
\hline
\end{tabular}

Em que: $\mathrm{SA}=$ sistema de amostragem simulado com dados de 13 parcelas, $\hat{b}_{0}, \hat{b}_{1}, \hat{b}_{2}$ e $\hat{b}_{3}=$ parâmetros estimados empregando-se dados de 13 parcelas, $\mathrm{s}(\mathrm{h} \hat{\mathrm{h}})$ e $\mathrm{r}(\mathrm{h} \hat{\mathrm{h}})=$ erro padrão residual e correlação linear, ambas estatísticas obtidas empregando-se dados de 33 parcelas.

(a)

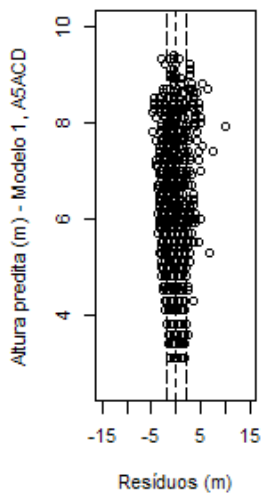

(e)

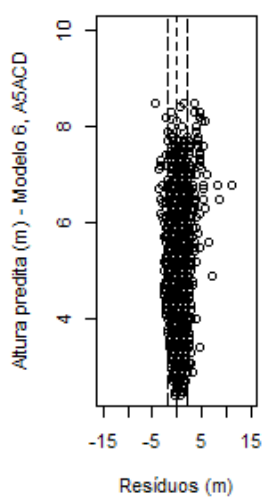

(b)

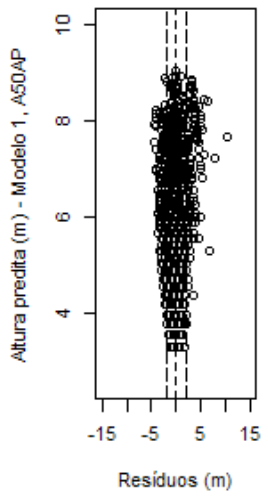

(f)

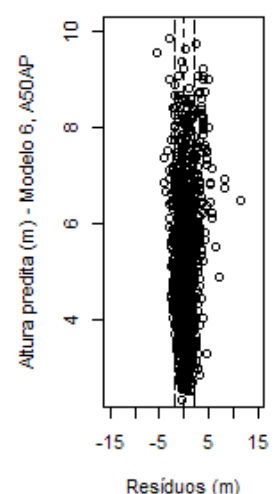

(c)

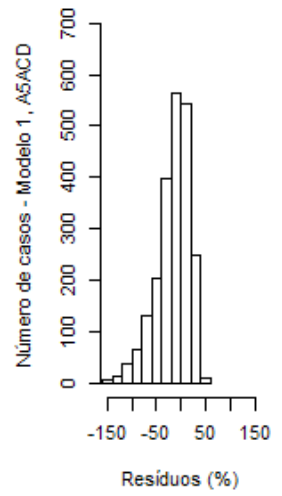

(g)

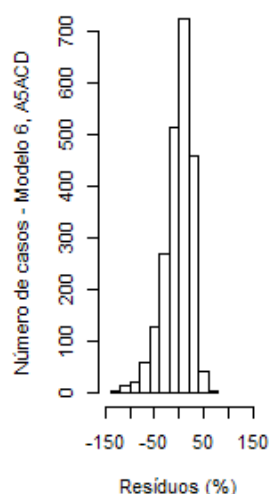

(d)

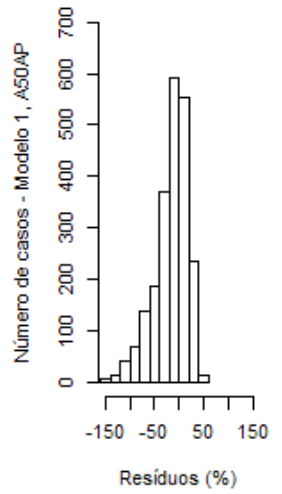

( h )

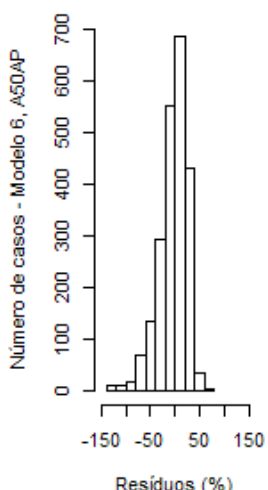

Figura 1. Distribuição de resíduos em relação à altura predita e histograma dos resíduos percentuais dos modelos 1 e 6, utilizando os sistemas A5ACD e A50AP. 
Empregando-se o sistema A50AP, nota-se que a distribuição diamétrica apresentou tendência de "J invertido" (Tabela 3 e Figura 3a), característico de floresta inequiânea tendendo ao equilíbrio (Felfili \& Silva-Junior, 1988; Fidelis \& Godoy, 2003; Assunção \& Felfili, 2004, Gomide et al., 2009; Giácomo et al., 2013; Sanqueta et al., 2013). A variável altura, por ter uma distribuição com o maior número de árvores (88,9\%) ocorrendo entre 2 e 8 m (Tabela 3 e Figura $3 b$ ), mostra uma tendência parecida com o que se espera de

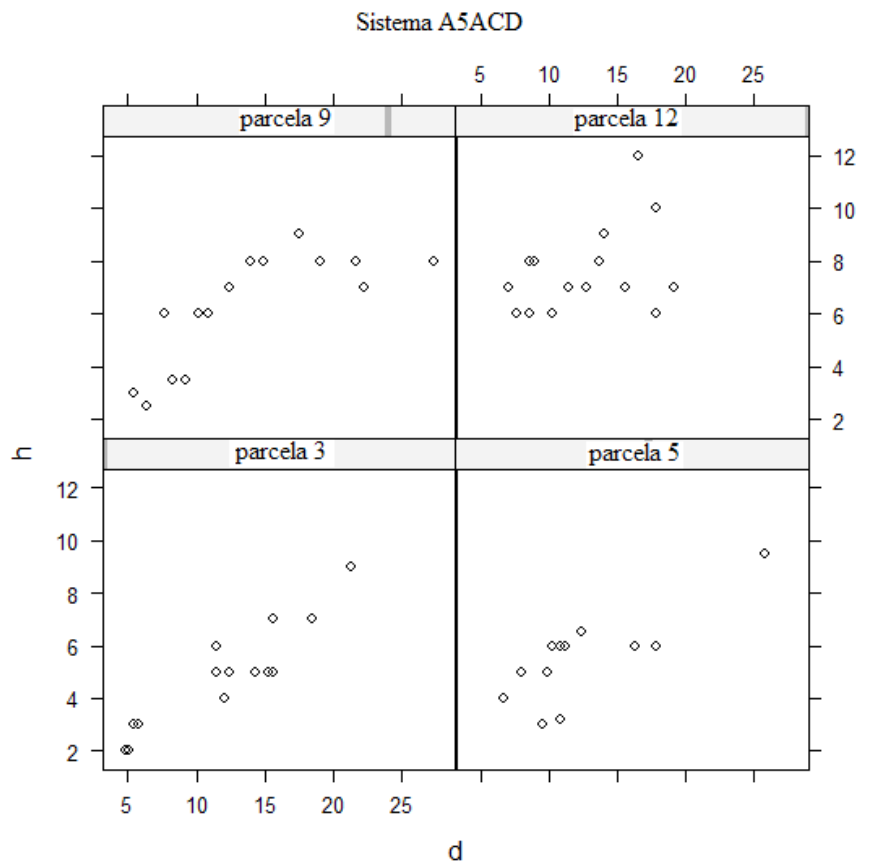

um cerrado sensu stricto com árvores baixas (Fidelis \& Godoy, 2003; Assunção \& Felfili, 2004, Ribeiro \& Walter, 2008; Giácomo et al., 2013), e também semelhante à distribuição observada em outros tipos de formações florestais inequianeas (Gomide et al., 2009 e Sanqueta et al., 2013). Na Tabela 4 é apresentado um resumo descritivo dos dados, indicando que o diâmetro tem maior variabilidade, seguido do número de árvores e da altura. O diâmetro médio quadrático, expressivamente, apresentou a menor variabilidade.

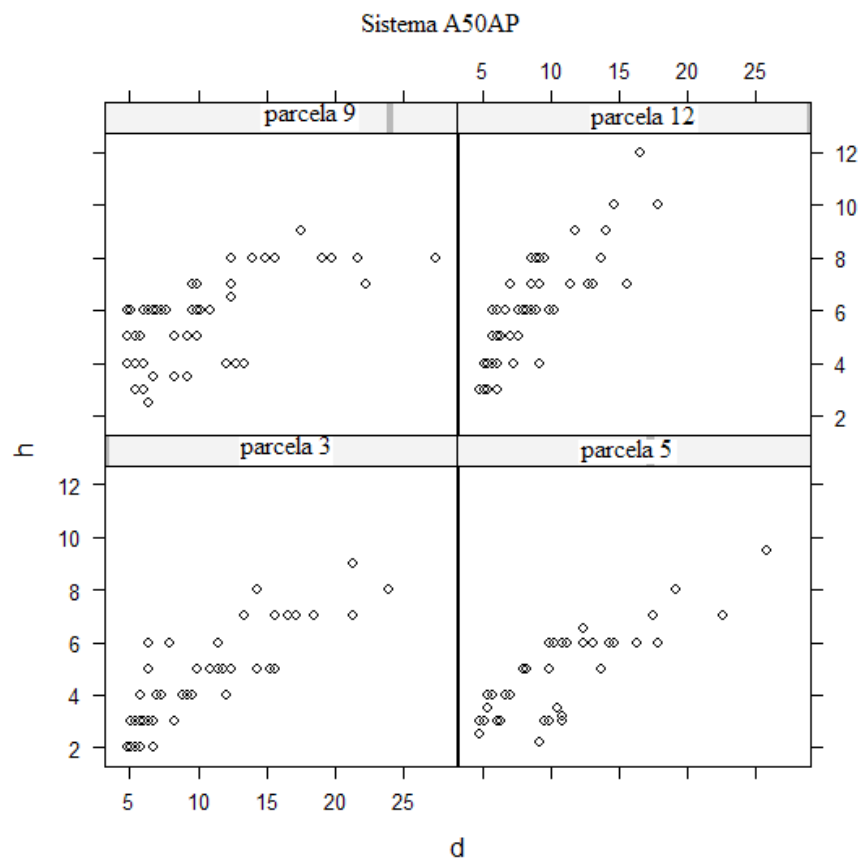

Figura 2. Altura (h) em relação ao diâmetro (d) para quatro parcelas selecionadas ao acaso dentre 13 parcelas nos sistemas de amostragens A5ACD e A50AP em cerrado sensu stricto tocantinense.

Tabela 3. Distribuição de frequência das 650 árvores amostradas pelo sistema A50AP em 13 parcelas distribuídas sistematicamente em uma área de cerrado sensu stricto tocantinense.

\begin{tabular}{|c|c|c|c|c|c|c|c|c|}
\hline \multirow{2}{*}{\multicolumn{2}{|c|}{$\begin{array}{c}\text { Classe de diâmetro } \\
(\mathrm{cm})\end{array}$}} & \multicolumn{6}{|c|}{ Classe de altura (m) } & \multirow{3}{*}{$\begin{array}{r}\text { Total } \\
388\end{array}$} \\
\hline & & \multirow{2}{*}{$\begin{array}{c}3(1) \\
177\end{array}$} & \multirow{2}{*}{$\begin{array}{c}5(2) \\
162\end{array}$} & \multirow{2}{*}{$\begin{array}{c}7(3) \\
47\end{array}$} & \multirow{2}{*}{$\frac{9(4)}{2}$} & \multirow[t]{2}{*}{$11(5)$} & \multirow[t]{2}{*}{$13(6)$} & \\
\hline (1) & 7,5 & & & & & & & \\
\hline (2) & 12,5 & 24 & 53 & 40 & 9 & 6 & & 132 \\
\hline \multirow[t]{2}{*}{ (3) } & 17,5 & 4 & 21 & 50 & 28 & 20 & 7 & 130 \\
\hline & Total & 205 & 236 & 137 & 39 & 26 & 7 & 650 \\
\hline
\end{tabular}


A

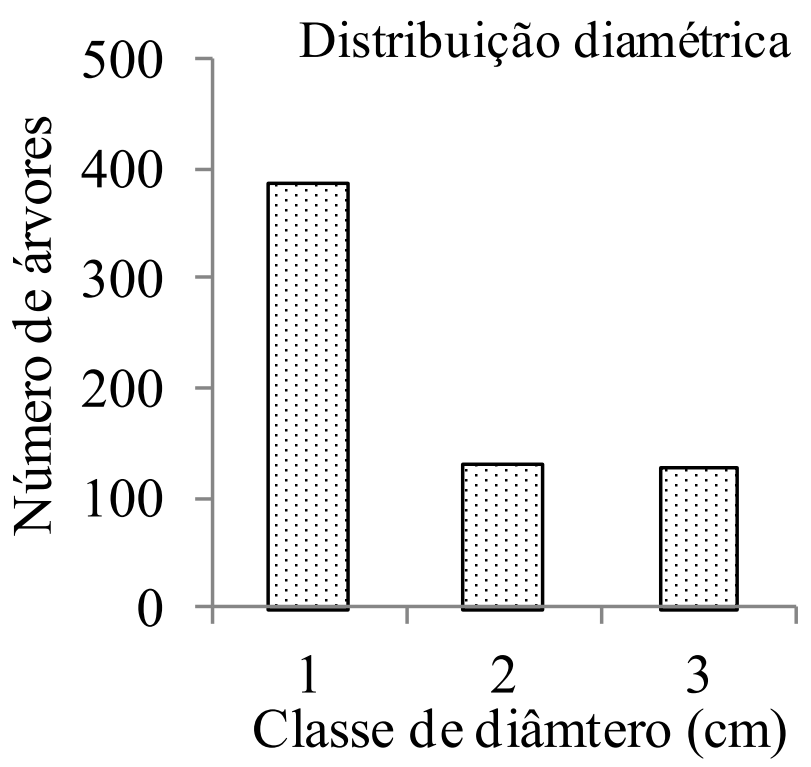

$\mathrm{B}$

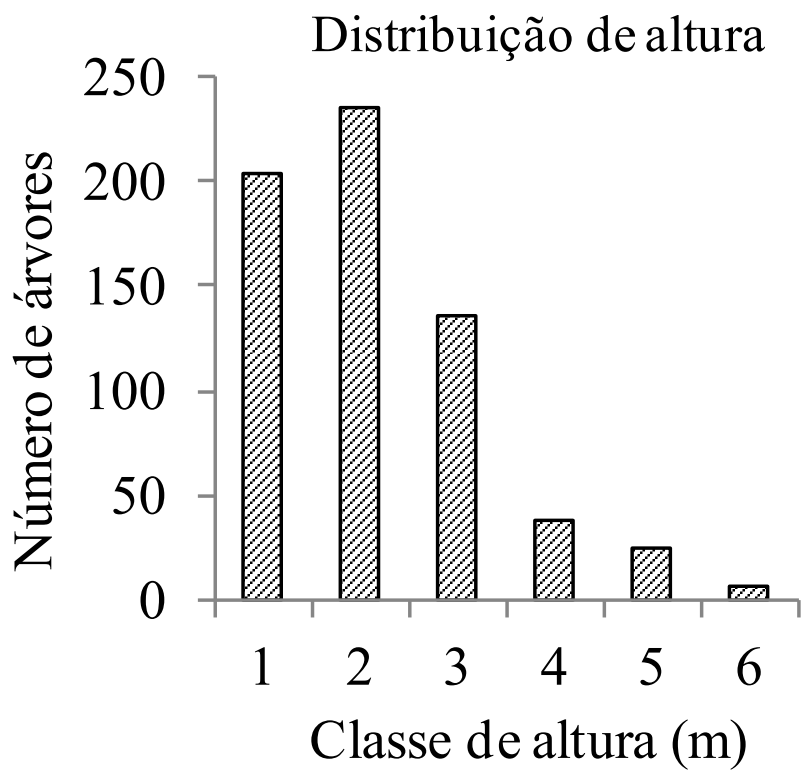

Figura 3. Gráficos de distribuição diamétrica (a) e de distribuição de altura (b) das 650 árvores amostradas pelo sistema A50AP em 13 parcelas distribuídas sistematicamente em uma área de cerrado sensu stricto tocantinense.

Tabela 4. Estatísticas descritivas das 650 árvores amostradas pelo sistema A50AP em 13 parcelas distribuídas sistematicamente em uma área de cerrado sensu stricto tocantinense.

\begin{tabular}{ccccc}
\hline Variável & Mínimo & Máximo & Média & $\begin{array}{c}\text { Coeficiente } \\
\text { de variação }\end{array}$ \\
d & 4,77 & 38,52 & 10,81 & 57,12 \\
$\mathbf{h}$ & 2,00 & 19,00 & 5,83 & 41,02 \\
$\mathbf{N}$ & 690 & 3.260 & $1.296,9$ & 52,50 \\
$\mathbf{d q}$ & 8,85 & 16,02 & 12,10 & 16,18 \\
\hline
\end{tabular}

Sendo $\mathrm{d}=$ diâmetro, $\mathrm{h}=$ haltrua, $\mathrm{N}=$ número de árvores por ha, $\mathrm{dq}=$ diâmetro médio quadrático $(\mathrm{cm})$.

Selecionados o melhor modelo hipsométrico e o sistema de amostragem de dados de relação hipsométrica, os quais se referem ao modelo 6 utilizando-se os dados obtidos por meio do sistema A50AP, é necessário, ainda, decidir sobre o uso desta base de dados com ou sem agrupamento. Neste sentido, decidiu-se avaliar o ajuste do modelo 1 com dados agrupados por parcela e o ajuste do modelo 6 com dados agrupados por classe de diâmetro, frente ao ajuste sem agrupamento que apresenta todas as informações de relação hipsométrica em um único lote de dados. Ressalta-se que a inclusão do modelo 1, com ajuste por parcela, se justifica por ser necessário comparar o seu desempenho com o ajuste do modelo 6 por classe diamétrica. Assim, utilizandose dados de 650 árvores obtidos em 13 parcelas com o sistema A50AP, foram estimadas as seguintes equações referentes aos modelos 1 e 6, conforme notação utilizada para emprego do teste $\mathrm{F}_{(\mathrm{Ho})}$ por Leite \& Andrade (2003):

Modelo 1, com a relação funcional: $\operatorname{Ln}(h)=f\left(\frac{1}{d}\right)$

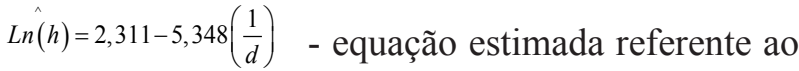
modelo 1 reduzido.

$$
\hat{\operatorname{Ln}(h)}=\hat{b}_{1} D_{1}+\ldots+\hat{b}_{13} D_{13}+\hat{b}_{14}\left(\frac{D_{1}}{d}\right)+\ldots+\hat{b}_{26}\left(\frac{D_{13}}{d}\right) \text { - equação }
$$

estimada referente ao modelo 1 completo.

Em que:

$\left[\begin{array}{lll}\mathrm{D}_{1} \mathrm{D}_{2} \mathrm{D}_{3} \ldots \mathrm{D}_{13}\end{array}\right]=\left[\begin{array}{lllll}1 & 0 & 0 & \ldots & 0\end{array}\right]$ para a parcela 1 ;

$\left[\begin{array}{lll}D_{1} D_{2} D_{3} \ldots & D_{13}\end{array}\right]=\left[\begin{array}{lllll}0 & 1 & 0 & \ldots & 0\end{array}\right]$ para a parcela 2 ;

$\left[\begin{array}{lllll}D_{1} & D_{2} D_{3} & \ldots & D_{13}\end{array}\right]=\left[\begin{array}{lllll}0 & 0 & 1 & \ldots & 0\end{array}\right]$ para a parcela 3 ;

$\left[\mathrm{D}_{1} \mathrm{D}_{2} \mathrm{D}_{3} \ldots \mathrm{D}_{13}\right]=\left[\begin{array}{lllll}0 & 0 & 0 & \ldots & 1\end{array}\right]$ para a parcela 13.

\section{Modelo 6, com a relação funcional:}

$$
\operatorname{Ln}(h)=f\left[\operatorname{Ln}(N) ; \operatorname{Ln}\left(\frac{d q}{d}\right) ; \frac{1}{d}\right. \text {. }
$$

$\hat{\operatorname{Ln}(h)}=0,44130+0,24389 \operatorname{Ln}(N)+0,22785 \operatorname{Ln}\left(\frac{d q}{d}\right)-3,66637\left(\frac{1}{d}\right)$ equação estimada referente ao modelo 6 reduzido. 


$$
\begin{aligned}
& \operatorname{Ln}(h)=\hat{b}_{1} D_{1}+\hat{b}_{2} D_{2}+\hat{b}_{3} D_{3}+\hat{b}_{4} D_{1} \operatorname{Ln}(N)+\hat{b}_{5} D_{2} \operatorname{Ln}(N) \\
& +\hat{b}_{6} D_{3} \operatorname{Ln}(N)+\hat{b}_{7} D_{1} \operatorname{Ln}\left(\frac{d q}{d}\right)+\hat{b}_{8} D_{2} \operatorname{Ln}\left(\frac{d q}{d}\right)+\hat{b}_{9} D_{3} \operatorname{Ln}\left(\frac{d q}{d}\right) \\
& +\hat{b}_{10} D_{1}\left(\frac{1}{d}\right)+\hat{b}_{11} D_{2}\left(\frac{1}{d}\right)+\hat{b}_{12} D_{3}\left(\frac{1}{d}\right)
\end{aligned}
$$

equação estimada referente ao modelo 6 completo.

Em que:

$\left[\mathrm{D}_{1} \mathrm{D}_{2} \mathrm{D}_{3} \ldots \mathrm{D}_{13}\right]=\left[\begin{array}{lllll}1 & 0 & 0 & \ldots & 0\end{array}\right]$ para a classe de diâmetro $1(\mathrm{~d} \leq 10 \mathrm{~cm}-\mathrm{cd} 1)$;

$\left[\mathrm{D}_{1} \mathrm{D}_{2} \mathrm{D}_{3} \ldots \mathrm{D}_{13}\right]=\left[\begin{array}{llll}0 & 1 & 0 & \ldots\end{array}\right]$ para a classe de diâmetro $2(10<\mathrm{d} \leq 15 \mathrm{~cm}-\mathrm{cd} 2)(\mathrm{cd} 1)$;

$\left[\mathrm{D}_{1} \mathrm{D}_{2} \mathrm{D}_{3} \ldots \mathrm{D}_{13}\right]=\left[\begin{array}{llll}0 & 0 & 1 & \ldots\end{array}\right]$ para a classe de diâmetro $3(\mathrm{~d}>15 \mathrm{~cm}-\mathrm{cd} 3)$.

$\mathrm{Na}$ decisão entre modelo reduzido e completo, considerou-se a estatística $\mathrm{F}_{(\mathrm{Ho})}$ apresentada na Tabela 5, a qual foi obtida com os dados das 650 árvores de 13 parcelas. O resultado foi significativo ao nível de $5 \%$ com valores de $14,4^{*}$ e $5,1^{*}$ para os modelos 1 e 6 , respectivamente. Isto indica que é mais apropriado ajustar o modelo 1 por parcela e o modelo 6 por classe de diâmetro ao invés de um único ajuste destes modelos sem agrupamento.

Tabela 5. Resultados estatísticos para os modelos hipsométricos 1 e 6, reduzido e completo, utilizando-se a base de dados com

\begin{tabular}{|c|c|c|c|c|}
\hline Modelo & $\mathrm{s}(\hat{\mathrm{hh}}) \%$ & $\mathrm{~s}(\mathrm{hh}) \mathbf{m}$ & $\mathrm{r}(\hat{\mathrm{hh}}) \%$ & $\mathrm{~F}_{\left(\mathrm{H}_{0}\right)}$ \\
\hline 1 - Completo (ajuste por parcela) & 24,13 & 1,404 & 72,03 & \multirow{2}{*}{14,4} \\
\hline 1 - Reduzido & 27,70 & 1,612 & 66,29 & \\
\hline $\begin{array}{l}6 \text { - Completo (ajuste por classe de } \\
\text { diâmetro) }\end{array}$ & 26,81 & 1,562 & 64,48 & \multirow{2}{*}{$5,1^{*}$} \\
\hline 6 - Reduzido & 26,76 & 1,559 & 65,27 & \\
\hline
\end{tabular}
13 parcelas empregando o sistema A50AP em cerrado sensu stricto tocantinense.

"significativo ao nível de 0,05 , Em que: $\mathrm{F}_{(\mathrm{Ho})}=$ estatística utilizada para decisão sobre usar modelo completo ou reduzido, utilizando dados de 650 árvores; $s(h \hat{h})$ e $r(h \hat{h})=$ erro padrão residual e correlação linear, ambas estatísticas obtidas empregando-se os dados restantes das árvores que não foram empregadas no ajuste.

Na Tabela 5 são apresentados os resultados de s(hĥ) e $\mathrm{r}(\mathrm{h} \hat{\mathrm{h}})$ para os modelos 1 e 6 (reduzido e completo), cujas estatísticas foram obtidas com os dados restantes de árvores das 13 parcelas, diferentes das 650 árvores empregadas no ajuste dos modelos 1 e 6 e no cálculo de $\mathrm{F}_{(\mathrm{Ho})}$. Nota-se que o modelo 1 completo (ajuste por parcela) apresentou resultados, expressivamente, melhores que os demais. Tal superioridade se repetiu na distribuição de resíduos (Figura 4), onde houve a melhor dispersão. Portanto, em áreas de cerrado sensu stricto do Tocantins, modelos hipsométricos locais tem que ser ajustados por parcela, pois são superiores até mesmo aos modelos regionais ajustados por classe diamétrica.
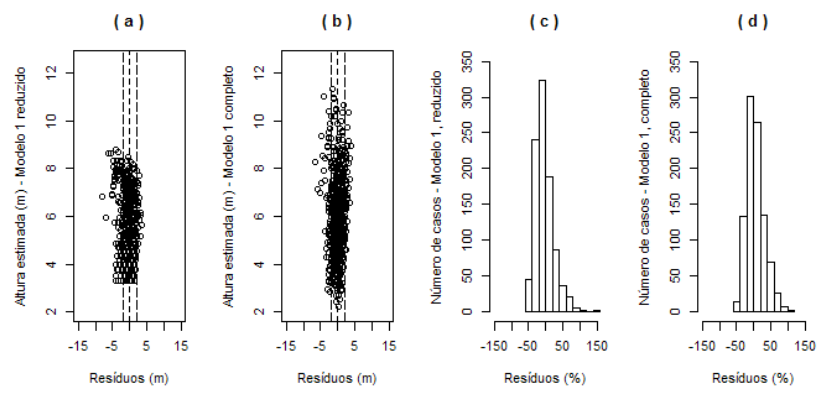

(e)

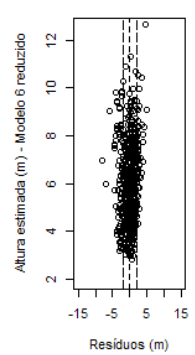

(f)

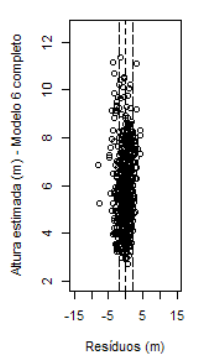

(g)

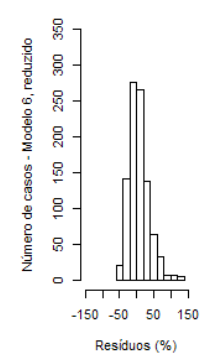

(h)

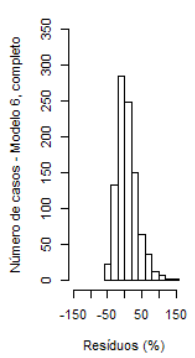

Figura 4. Distribuição de resíduos em relação à altura e histograma dos resíduos para os modelos 1 e 6 , reduzido e completo, ajustados com 650 árvores amostradas pelo sistema A50AP e aplicados na base de dados com o restante das árvores, ambas obtidas nas 13 parcelas em cerrado sensu stricto tocantinense.

Quanto ao modelo 6, apesar do teste $\mathrm{F}_{(\mathrm{Ho})}=5,1^{*}$ indicar ser mais apropriado o seu ajuste por classe de diâmetro

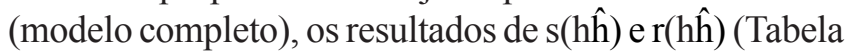
3) juntamente com a distribuição de resíduos (Figura $4 \mathrm{e}$ versus $4 \mathrm{f}$ e $4 \mathrm{~g}$ versus $4 \mathrm{~h}$ ), indicam haver pouca inferioridade deste em relação ao seu modelo reduzido. Portanto, ajustar modelos hipsométricos regionais por classe de diâmetro resulta em pouco ganho estatístico, comparado ao seu ajuste utilizando-se um só lote de dados, ou seja, o modelo regional completo por classe de diâmetro tem poucas vantagens em relação ao modelo regional reduzido. Este resultado também foi obtido por Andrade et al. (2006) para Mata Atlântica, onde demonstraram que o ajuste de modelos hipsométricos regionais empregando todas as informações sem agrupamento é preferível ao ajuste empregando dados de relação hipsométrica agrupados por classe de diâmetro. 
A dispersão obtida (Figura 5), separadamente, para as classes de diâmetro $1(\mathrm{~d} \leq 10 \mathrm{~cm}-\mathrm{cd} 1)$ e $2(10<$ $\mathrm{d} \leq 15 \mathrm{~cm}-\mathrm{cd} 2)$ não evidenciam tendência de dados para haver um bom ajuste de equações hipsométricas, referentes ao ajuste do modelo 6 . Somente na classe de diâmetro $3(\mathrm{~d}>15 \mathrm{~cm}-\mathrm{cd} 3)$ é que se observa uma dispersão favorável ao ajuste deste modelo. Por outro lado, quando se inclui todos os dados em um só lote, independentemente da classe de diâmetro, observa-se que a dispersão dos dados favorece o ajuste do modelo 6 na forma de modelo reduzido. Por isso, os resultados foram semelhantes entre modelo reduzido e completo (Tabela 5) e a distribuição de resíduos (Figura 4). Portanto, em áreas de cerrado sensu stricto do Tocantins, modelos hipsométricos regionais podem ser ajustados na forma reduzida sem ter os dados agrupados por classe de diâmetro.

Selecionou-se portanto, o modelo 1 com ajuste por parcela (modelo completo) e o modelo 6 com ajuste utilizando todas as informações em um único lote de dados (modelo reduzido), cujos dados foram obtidos pela amostragem das 50 primeiras árvores de cada uma das 13 parcelas. Deve-se destacar que o modelo 6 reduzido exigiu um único ajuste, em contraste ao observado para o modelo 1 completo que exigiu o ajuste de treze equações, sendo uma por parcela. Assim, a partir deste trabalho, observa-se a grande importância em se conduzir estudos para identificar a melhor estratégia de agrupamento de dados visando o ajuste de modelos hipsométricos regionais em cerrado sensu stricto no Tocantins. Como opções, pode-se considerar o ajuste utilizando dados de relação hipsométrica agrupados por espécie, por grupos de espécies, por local, ou ainda, por grupo de família botânica, conforme feito por Durlo \& Denardi (1998), Batista et al. (2001), Bartoszeck et al. (2004) e Sanqueta et al. (2013).

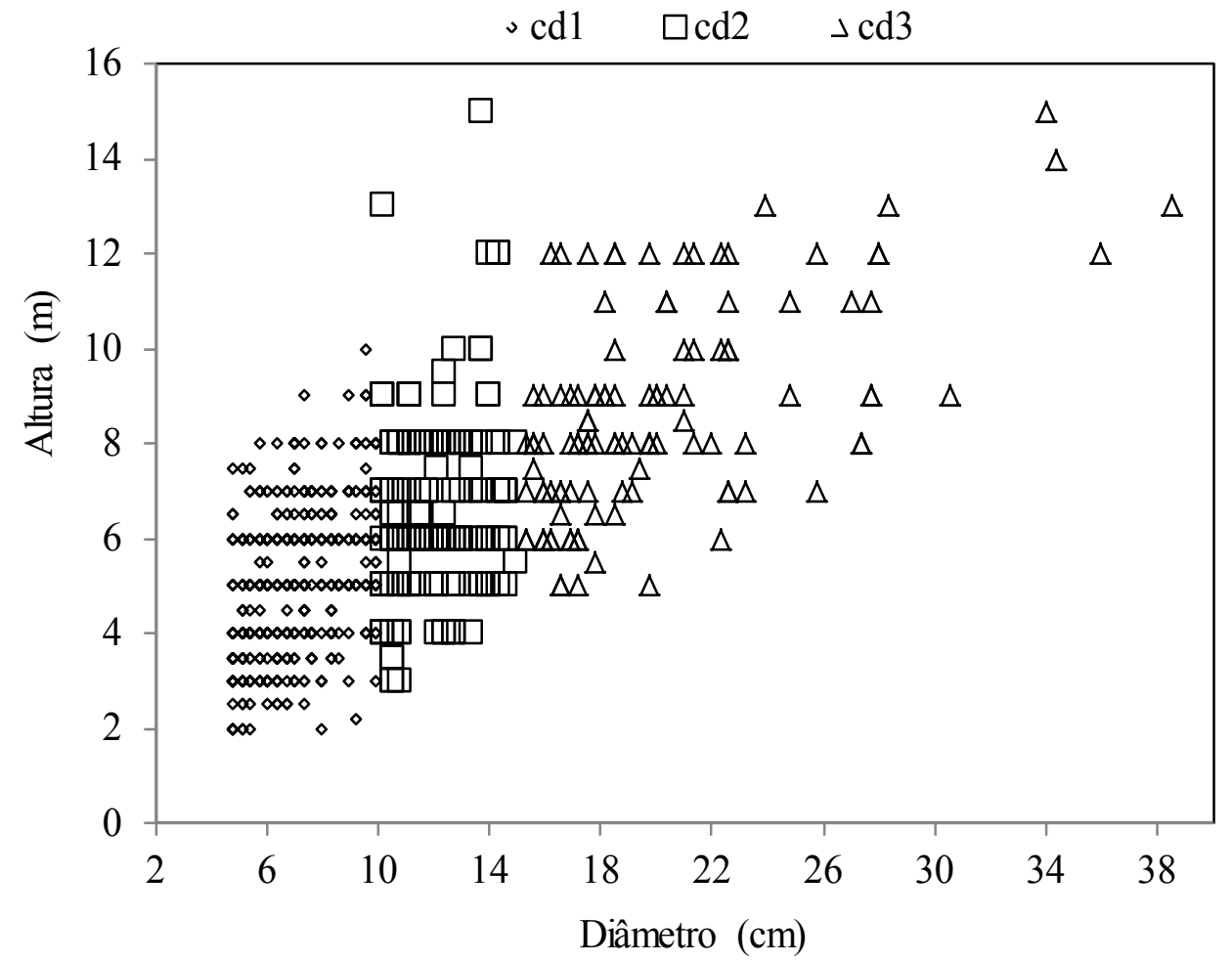

Figura 5. Altura em relação ao diâmetro para as classes 1, 2 e $3(\operatorname{cd} 1$, cd2 e cd3) no sistema de amostragem A50AP, utilizando-se a base de dados com 650 árvores de 13 parcelas, em cerrado sensu stricto tocantinense. 
É importante ressaltar que a dispersão dos resíduos e resultados dos critérios estatísticos obtidos para cerrado sensu stricto neste trabalho são coerentes com o que se esperava dentre as características de floresta inequiânea, semelhante ao observado por Durlo e Denardi (1998), Batista et al. (2001), Andrade et al. (2006), Costa et al (2012) e Sanqueta et al. (2013). Os valores de $\mathrm{s}(\mathrm{h} \hat{\mathrm{h}})$ e $\mathrm{r}(\mathrm{h} \hat{\mathrm{h}})$ variaram entre 15,9 a $19,4 \% \mathrm{e}$ 55 a $92 \%$, respectivamente. Deve-se observar, ainda, que caracterizar a relação hipsométrica em área de cerrado é mais complexo que em outro tipo de floresta nativa. Conforme Costa et al. (2012), que trabalharam com candeia em diferentes fisionomias de floresta no sul de Minas Gerais, $\mathrm{o} \overline{\mathrm{R}}^{2}$ obtido para floresta foi bem superior ao obtido para cerrado $(0,82$ versus 0,30$)$, retratando assim pior caracterização da relação hipsométrica em formação florestal de cerrado.

De maneira geral, a complexidade do ajuste de modelos hipsométricos em mata nativa é bem maior que em plantios, dado à grande variabilidade dos dados de relação hipsométrica neste tipo de formação florestal. Para melhor verificar isto, conforme os trabalhos feitos por Barros et al. (2002), Ribeiro et al. (2010), Mendonça et al. (2011), Rossi et al. (2011) e Araújo et al. (2012), os valores de $\mathrm{s}(\mathrm{h} \hat{\mathrm{h}})$ e $\mathrm{r}(\mathrm{h} \hat{\mathrm{h}})$ variaram entre 6,3 a $24,8 \%$ e 56 a $98 \%$, com a maioria sendo inferior a $10 \%$ e superior a $80 \%$, respectivamente. Isso é apenas uma amostra que permite evidenciar a grande complexidade e dificuldade sobre o uso de equações hipsométricos em mata nativa, principalmente em áreas de cerrado. Apesar disso, em mata nativa, recomenda-se conduzir inventários florestais empregando-se equações hipsométricas.

\section{Conclusões}

A relação funcional linear $\operatorname{Ln}(h)=f\left[\operatorname{Ln}(N) ; \operatorname{Ln}\left(\frac{d q}{d}\right) ; \frac{1}{d}\right]$ foi a mais adequada para caracterizar a relação hipsométrica em inventários florestais de cerrado sensu stricto tocantinense e deve ser ajustada com dados obtidos nas 50 primeiras árvores de cada parcela, sem agrupamento por classe de diâmetro.

Devem-se conduzir outros estudos para identificar a melhor estratégia de ajuste dos modelos hipsométricos em outras regiões em cerrado sensu stricto tocantinense.

\section{Referências}

ANDRADE, V. C. L.; MARTINS, S. V.; CALEGARIO, N.; CHICHORRO, J. F. Influência de três sistemas de amostragem na estimativa da relação hipsométrica e do volume de árvores em um fragmento de Mata Atlântica. Scientia Forestalis, Piracicaba, n. 70, p. 31-37, 2006

ARAÚJO, E. J. G.; PELISSARI, A. L.; DAVID, H. C.; MIRANDA, R. O. V.; SCOLFORO, J. R. S.; PELLICO-NETO, S.; MORAIS, V. A. Relação hipsométrica para candeia (Eremanthus erythropappus) com diferentes espaçamentos de plantio em Minas Gerais, Brasil. Pesquisa Florestal Brasileira, Colombo, v. 32, n. 71, p. 257-268, 2012. DOI: $10.4336 / 2012$.pfb.32.71.257

ASSUNÇÃO, S. L.; FELFILI, J. M. Fitossociologia de um fragmento de cerrado sensu stricto na APA do Paranoá, DF, Brasil. Acta Botânica Brasilica, São Paulo, v. 18, n. 4, p. 903-909, 2004. DOI: $10.1590 / \mathrm{S} 0102-33062004000400021$

ATLAS DO TOCANTINS: subsídios ao planejamento da gestão territorial. 5. ed. Palmas: Secretaria do Planejamento e Meio Ambiente, 2008. 62 p.

BARROS, D. A.; MACHADO, S. A.; ACERBI JÚNIOR, F. W.; SCOLFORO, J. R. S. Comportamento de modelos hipsométricos tradicionais e genéricos para plantações de Pinus oocarpa em diferentes tratamentos. Boletim de Pesquisa Florestal, Colombo, n. 45 , p. $03-28,2002$.

BATISTA, J. L. F.; COUTO, H. T. Z.; MARQUESINI, M. Desempenho de modelos de relações hipsométricas: estudo em três tipos de florestas. Scientia Forestalis, Piracicaba, n. 60, p. 149-163, 2001.

BARTOSZECK, A. C. P. S.; MACHADO, S. A.; FIGUEIREDO FILHO, A.; OLIVEIRA, E.B. Dinâmica da relação hipsométrica em função da idade, do sítio e da densidade inicial de povoamentos de bracatinga da região metropolitana de Curitiba, PR. Revista Árvore, Viçosa, MG, v. 28, n. 4, p. 517-533, 2004. DOI: 10.1590/ S0100-67622004000400006

CALDEIRA, M. V. W.; SCHUMACHER, M. V.; SCHEEREN, L. W.; WATZLAWICK, L. F. Relação hipsométrica para Araucaria angustifolia (Bert.) O. Ktze na Região Oeste do Estado do Paraná. Revista Acadêmica: ciências agrárias e ambientais, Curitiba, v. 1, n. 2, p. 79-88, 2003.

CARDOSO, D. J.; MACHADO, S. do A.; ROSOT, N. C.; EMERECIANO, D. B. Avaliação da influência dos fatores idade e sítio na relação hipsométrica para Pinus taeda nas regiões central e Sudoeste do estado do Paraná. Floresta, Curitiba, v. 19, n. 1/2, p. 96-115, 1989

COSTA, M. P.; PEREIRA, J. A. A.; BENICIO, M. H. M.; SOUZA, H.; FONTES, M. A. L.; GARCIA, P. O. Alometria e arquitetura de Copaifera langsdorffii (desf.) kuntze (fabaceae) em fitofisionomias neotropicais no sul de Minas Gerais. Ciência Florestal, Santa Maria, RS, v. 22, n. 2, p. 223-240, 2012. DOI: 10.5902/198050985729

COUTINHO, L. M. O conceito de cerrado. Revista Brasileira de Botânica, São Paulo, v. 1, n. 1, p. 17-23, 1978. 
DURLO, M. A.; DENARDI, L. Morfometria de Cabralea canjerana, em mata secundaria nativa do Rio Grande do Sul. Ciência Florestal, Santa Maria, RS, v. 8, n. 1, p. 55-66, 1998.

EITEN, G. Delimitação do conceito de Cerrado. Arquivos do Jardim Botânico, Rio de Janeiro, v. 21, p. 125-134, 1977.

FELFILI, J. M.; SILVA-JUNIOR, M. C. Distribuição dos diâmetros numa faixa de cerrado na fazenda agua limp a (fal) em brasilia-df. Acta Botânica Brasilica, Feira de Santana, v. 2, n. 1/2, p. 85-104, 1988. DOI: $10.1590 / \mathrm{S} 0102-33061988000100005$

FIDELIS, A. T.; GODOY, S. A. P. Estrutura de um cerrado strico sensu na gleba cerrado Pé-De-Gigante, Santa Rita do Passa Quatro, SP. Acta Botânica Brasilica, São Paulo, v. 17, n. 4, p. 531-539, 2003. DOI: $10.1590 / \mathrm{S} 0102-33062003000400006$

GIÁCOMO, R. G.; CARVALHO, D. C.; PEREIRA, M. G.; SOUZA, A. B.; GAUI, T. D. Florística e fitossociologia em áreas de campo sujo e cerrado sensu stricto na estação ecológica de pirapitinga-MG. Ciência Florestal, Santa Maria, RS, v. 23, n. 1, p. 29-43, 2013. DOI: 10.5902/198050988437

GOMIDE, L. R.; SCOLFORO, J. R. S.; OLIVEIRA, A. D. Análise das estruturas diamétrica e hipsométrica de fragmentos florestais localizados na bacia do rio São Francisco, em Minas Gerais, Brasil. Floresta, Curitiba, v. 39, n. 2, p. 239-251, 2009. DOI: 10.5380/ rf.v39i2.14553

LEITE, H. G.; ANDRADE, V. C. L. Importância das variáveis altura dominante e altura total em equações hipsométricas e volumétricas. Revista Árvore, Viçosa, MG, v. 27, n. 3, p. 301-310, 2003.

MACHADO, S. A.; BAILEY, R. L.; BASSO, S. F.; BEVILACQUA, V. G. Análise do comportamento da relação hipsométrica com respeito a idade para plantações de Pinus elliottii no Estado do Paraná. Cerne, Lavras, v. 1, n. 1, p. 5-12, 1994.

MACHADO, S. A.; NASCIMENTO, R. G. M.; AUGUSTYNCZIK, A. L. D.; SILVA, L. C. R.; FIGURA, M. A.; PEREIRA, E. M.; TÉO, S. J. Comportamento da relação hipsométrica de Araucaria angustifolia no capão da Engenharia Florestal da UFPR. Pesquisa Florestal Brasileira, Colombo, n. 56, p. 5-16, 2008.
MENDONÇA, A. R.; CALEGARIO, N.; SILVA, G. F.; BORGES, L. A. C.; CARVAlHO, S. P. C. Modelos hiposométricos e de crescimento em altura das árvores dominantes e codominates para Pinus caribaea var. hondurensis. Scientia Forestalis, Piracicaba, v. 39, n. 90, p. 151-160, 2011.

REGAZZI, A. J. Teste para verificar a identidade de modelos de regressão. Pesquisa Agropecuária Brasileira, Colombo, v. 31, n. 1, p. 1-17, 1996.

RIBEIRO, A.; FERRAZ FILHO, A. C.; MELlO, J. M. de; FERREIRA, M. Z.; LISBOA, P. M. M.; SCOLFORO, J. R. S. Estratégias e metodologias de ajuste de modelos hipsométricos em plantios de Eucalyptus sp. Cerne, Lavras, v. 16, n. 1, p. 22-31, 2010.

RIBEIRO, J. F.; WALTER, B. M. T. As principais fitofisionomias do Cerrado. In: SANO, S. M.; ALMEIDA. S. P.; RIBEIRO, J. F. (Ed.). Cerrado: ecologia e flora. Planaltina: Embrapa Cerrados, 2008. p. 153-212.

ROSSI, A. S.; DRESCHER, R.; PELISSARI, A. L.; LANSSANOVA, L. R. Relação hipsométrica e crescimento de Tectona grandis L.f. no município de Monte Dourado, Pará. Scientia Forestalis, Piracicaba, v. 39, n. 91, p. 301-307, 2011.

SANQUETA, C. R.; DALlA CORTE, A. P.; ROGLIN, A.; PIMENTEL, A. Relações diâmetro-altura para espécies lenhosas em um fragmento de Floresta Ombrófila Mista no Sul do Paraná. Iheringia: Série Botânica, Porto Alegre, v. 68, n. 1, p. 103-114, 2013.

SISTEMA Brasileiro de Classificação de Solos. Brasília, DF: Embrapa Produção de Informação; Rio de Janeiro: Embrapa Solos, 1999. 412 p.

SCHIMIDT, P. B. Determinação indireta da relação hipsométrica para povoamentos de Pinus taeda L. Floresta, Curitiba, v. 8, n. 1, p. 24-27, 1977.

SOARES, C. P. B; MARTINS, F. B.; LEITE JUNIOR, H. U.; SILVA, G. F.; FIGUEIREDO, L. T. M. Equações hipsométricas, volumétricas e de taper para onze espécies nativas. Revista Árvore, Viçosa, MG, v. 35, n. 5, p. 1039-1051, 2011.

TOCANTINS. Secretaria do Planejamento e Meio Ambiente. Projeto de Gestão Ambiental Integrada da Região do Bico do Papagaio. Zoneamento Ecológico-Econômico. Base de dados geográficos do norte do Tocantins. Palmas, 2005. 